\title{
Quote-part différenciée pour des préparations originales et des génériques
}

Gert Printzen ${ }^{a}$, Ernst Gähler ${ }^{b}$

a Dr, Membre du Comité central, responsable du domaine Produits thérapeutiques

b Dr, Vice-président de la FMH, responsable du domaine Tarifs et Conventions
Jusqu'à présent les génériques bénéficiaient d'une quote-part de $10 \%$ alors que les originaux pour lesquels il existe des génériques meilleurs marchés étaient soumis à une quote-part de $20 \%$. Depuis le $1^{\text {er }}$ juillet 2011, un générique peut désormais avoir une quote-part de $10 \%$ ou de $20 \%$. La loi stipule que «la quote-part s'élève à $20 \%$ des coûts dépassant la franchise pour les médicaments dont le prix maximal dépasse de plus de $20 \%$ la moyenne des prix maximaux du tiers le plus avantageux de tous les médicaments contenant la même substance active et figurant sur la liste des spécialités». La liste des génériques avec quote-part différenciée pour des préparations originales et des génériques est actualisée tous les mois par l'Office fédéral de la santé publique (OSFP); cette liste est disponible sous www.bag. admin.ch $\rightarrow$ Thèmes $\rightarrow$ Assurance-maladie $\rightarrow$ Tarifs et prix $\rightarrow$ Liste des spécialités $\rightarrow$ Index des répertoires de la LS.

La nouvelle réglementation complique la situation aussi bien pour les médecins que pour les patients; ainsi il n'est plus possible de prévoir quels seront les médicaments qui impliqueront une quotepart plus élevée et donc de faire en sorte que les patients évitent de s'y habituer.

Ce qui est gênant dans ce genre de réglementations, c'est qu'un médicament original et son générique peuvent provoquer des effets différents. Les deux produits contiennent la même substance active mais les originaux se distinguent des génériques dans leur composition générale; le générique n'est pas une copie de l'original. Le problème est par exemple très connu avec les médicaments pour épileptiques.

Cette ordonnance empêche d'adapter parfaitement un traitement médicamenteux aux besoins du patient. Synonyme d'une réduction massive de la liberté thérapeutique, elle se dresse en obstacle de la relation de confiance entre le médecin et son patient. Tout changement de médicament représente un risque pour le patient mais aussi pour le médecin. Nous savons que la galénique, le mode d'application, la taille et la couleur du comprimé sont des aspects tout aussi importants que l'économie de la substance active pure. Précisément chez les patients polymorbides, qui génèrent près de $80 \%$ des coûts, les changements fréquents de médicaments compromettent gravement la sécurité des patients.

Au-delà des préoccupations d'ordre purement médical, il faut aussi se poser la question de la compatibilité d'une ordonnance aussi compliquée - dont l'objectif est d'économiser des coûts - avec le quotidien d'un cabinet médical. Comment s'imagine-t-on sa mise en application? N'oublions pas que la charge administrative ne cesse d'augmenter!

En imposant de telles réglementations aux médecins et aux patients (de surcroît sans procédure d'audition préalable), le monde politique adopte une communication paradoxale alors qu'il souhaite en même temps que les médecins intensifient leur engagement en faveur de la sécurité des médicaments.

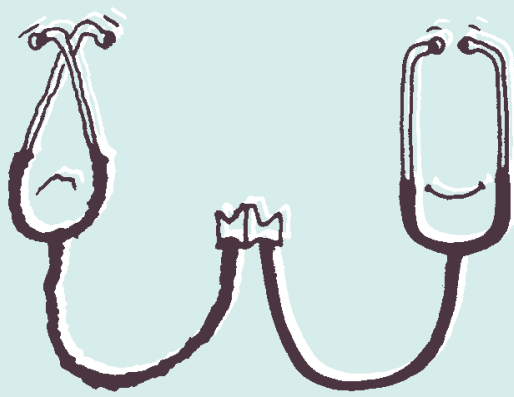

\section{Et soudain, vous aussi! Les crises touchent aussi les médecins.}

Acceptez de l'aide. Contactez ReMed.

Assistance téléphonique 24 h sur 24

0800073633 help@swiss-remed.ch

08000 ReMed www.swiss-remed.ch 